\title{
Detection of Casimir Radiation from Our Sun
}

\author{
Richard A. Hutchin \\ Optical Physics Company, Simi Valley, CA, USA \\ Email: rahutchin@opci.com
}

How to cite this paper: Hutchin, R.A. (2019) Detection of Casimir Radiation from Our Sun. Optics and Photonics Journal, 9, 141-154.

https://doi.org/10.4236/opj.2019.99013

Received: August 1, 2019

Accepted: September 9, 2019

Published: September 12, 2019

Copyright $\odot 2019$ by author(s) and Scientific Research Publishing Inc. This work is licensed under the Creative Commons Attribution International License (CC BY 4.0).

http://creativecommons.org/licenses/by/4.0/ (c) (i) Open Access

\begin{abstract}
This paper extends the previous experimental work on Planck's constant $h$ and the vacuum field, whose spectrum is determined by $h$. In particular it adds additional experimental evidence supporting temporal and spatial variations in the vacuum field, including the Sun as a source at 13 sigmas of certainty. The vacuum field has long been a mystery of physics, having enormous theoretical intensity set by Planck's constant $\mathrm{h}$ and yet no obvious physical effect. Hendrick Casimir first proposed that this form of E \& M radiation was real in 1948 and suggested an experiment to verify its existence. Over 50 experiments since then have confirmed that this vacuum radiation is real, is a form of electro-magnetic radiation, and varies in time and space over 10:1 in our laboratory compared to its standard QM spectrum. Two other authors have found the fine structure constant $\alpha$ (proportional to $1 / \mathrm{h}$ ) is varying across the cosmos at up to 4.2 sigma certainty. All these results suggest that the vacuum field (and thus $h$ ) varies in time and space. In a previous paper we reported our tunnel diode experimental results as well as the results of six other organizations (including German, Russian and US national labs).The six organizations reported sinusoidal annual variations of $1000-3000 \mathrm{ppm}$ (peak-to-valley) in the decay rates of 8 radionuclides over a 20 -year span, including beta decay (weak interaction) and alpha decay (strong interaction). All decay rates peaked in January-February and minimized in July-August without any candidate cause suggested. We confirmed that Planck's constant was the cause by verifying similar variations in Esaki tunnel diode current, which is purely electromagnetic. The combined data from previous strong and weak decays plus our own E \& M tunnel data showed similar magnitude and time phasing for strong, weak and $\mathrm{E} \& \mathrm{M}$ interactions, except that the tunnel diode temporal variations were $180 \mathrm{deg}$ out of phase-as we predicted. The logic for this 180 deg phase shift was straight forward. Radioactive decay and electron tunneling both have $\mathrm{h}$ in the denominator of the tunneling exponent, but tunnel diodes also have $\mathrm{h}^{2}$ in the numerator of the exponent due to the size of atoms being proportional to $h^{2}$. This extra $h^{2}$ makes the exponent proportional to $\mathrm{h}$ for electron tunneling instead of proportional to $1 / \mathrm{h}$
\end{abstract}


for strong and weak decay-shifting the annual oscillation for E \& M tunnel current by $180 \mathrm{deg}$. Radioactive decay had a maximum around January-February of each year and a minimum around July-August of each year. Tunnel current (the equivalent to radioactive decay rate) had the opposite-a minimum around January of each year and a maximum around July of each year. This predicted and observed sign flip in the temporal variations between radioactive decay and electron tunneling provides strong evidence that $h$ variations across the Earth's orbit are the cause of these annual cycles. In this paper we take the next step by verifying whether the Sun and a potential more distant cosmic source radiate the vacuum E \& M field, just as all stars generate massive amounts of regular E \& M radiation. We reprocessed two years of data, 6 million data points, from our tunnel diode experiment to search for day-night oscillations in tunnel current. Here we assume that the Earth would block the radiated vacuum field half of each day. Sun-locked signals have 365 cycles per year and cosmos locked signals have 366 cycles per year. With our two years of data, these two signals are separated by a null-signal, which is not locked to the Earth or to the cosmos-allowing us to clearly distinguish the solar and cosmic sources. 1) We found sun-locked variations in the vacuum field, peaking around local noon with $10^{-13}$ probability of false alarm. Other potential causes are carefully examined and ruled out. 2) We also found cosmos-locked variations in the vacuum field, peaking at the right ascension of the red super-giant star Betelgeuse with $10^{-7}$ probability of false alarm. Cosmos locked sources are easily distinguished from the solar source because they have one extra cycle per year, two extra cycles during the two years of the experiment. They are thus independent Fourier components, easily separated by a Fourier transform. Both of these high probability detections support that the vacuum field spectrum may vary in space and time and be enhanced by stellar sources.

\section{Keywords}

Planck's Constant, Variable Radioactive Decay Rate, Variable Plank's Constant, Solar Radiation, Casimir Radiation, Cosmic Red Shift

\section{Introduction}

This paper explores the nature of the Vacuum Field, which has been a mystery for 100 years. In first half of the $20^{\text {th }}$ century, many scientists thought the ground state energy of the E \& M field (called the vacuum state) was a mathematical result without physical meaning until Hendrik Casimir proposed an experimental test in 1948. This experimental work verified the existence of the vacuum field at power levels equal to the entire power generation of our Earth civilization in every square inch. The observed pressure in the Casimir experiments matched the terawatts $/ \mathrm{cm}^{2}$ of predicted E \& M power, but nothing was heated. The vacuum field is currently regarded to be a vastly intense form of E \& M radiation that creates pressure without heat.

In these Casimir experiments, the observed radiation pressure of 19 psi oc- 
curred because the intensity of the vacuum field varied more than 10:1 between the two plates compared to outside the plates. If the vacuum field can vary hugely in our labs, one might expect the vacuum field could also vary in space and time across the universe, and this research detects and reports such variations in our solar system and beyond.

Past research papers from three national labs and other groups showed annual variations in strong and weak radioactive decay rates without proposing a cause [1]-[10]. Our contribution was first to hypothesize that Planck's constant was the link between strong and weak interactions, suggesting that variations in $h$ might be responsible. We then tested this hypothesis using tunnel diodes-purely an E \& $M$ phenomenon-and found the same annual oscillation of similar magnitude except180 deg out of phase-as predicted and discussed below. A short summary of the data is given here, with the details in [11] and pictures of the hardware in Appendix, showing the design of the tunnel diode experiment.

Two cosmological studies are also referenced [12] [13], which show 3.6 and 4.2 sigma variations of the fine structure constant $\alpha$ with cosmic distance in a dipole mode with angle. Since the fine structure constant includes $h$ in its denominator, these data also support spatial variations in $h$ across the cosmos.

The ability for the vacuum field to vary in time and space by factors of 10 or more has been verified in our laboratories by many published experiments, all derived from Casimir's original paper in 1948 [14] [15]. The pressure created by the well-accepted variations in the vacuum field intensity has been refined to the point where this variable vacuum field even drives micro-machines [15]. Thus this research was trying to determine if the variations in the vacuum field we see in our labs are also present in space, and if so, to identify the sources of those variations.

In searching for potential sources of the vacuum field, we analyzed the previous tunnel diode data to look for potential vacuum field radiation coming from our Sun. We found a daily tunnel signal consistent with increased $h$ that peaked at noon each day. The probability of false alarm was about $10^{-13}$.

In addition, the same analysis found a radiation signal locked to the distant cosmos with a $10^{-7}$ probability of false alarm aligned in the direction of Betelgeuse, a red super-giant as bright as 55,000 suns. This star is predicted by astronomers to go supernova in about one million years.

We begin with a short summary of the previously reported time-varying radioactive decay and annual tunnel diode variations, and the logic that leads to the conclusion that variations in $\mathrm{h}$ are responsible.

Then the new data is presented along with its more detailed analysis, showing high probability detection of radiation from the sun and a separate cosmic source. In respect for Hendrik Casimir, who started the exploration of the vacuum field in 1948, we have called this new form of radiation, Casimir Radiation.

1) Time Varying Radioactive Decay Rate. 
Annual variations of 1000 - 3000 ppm (peak-to-valley) have been observed in the decay rates of 8 radionuclides over a 20 -year span by six organizations on three continents, including German, Russian and US national labs [1]-[10]. The decay modes affected included beta decay (weak interaction) and alpha decay (strong interaction). Table 1 summarizes the results and Figure 1 and Figure 2 show some of the experimental results.

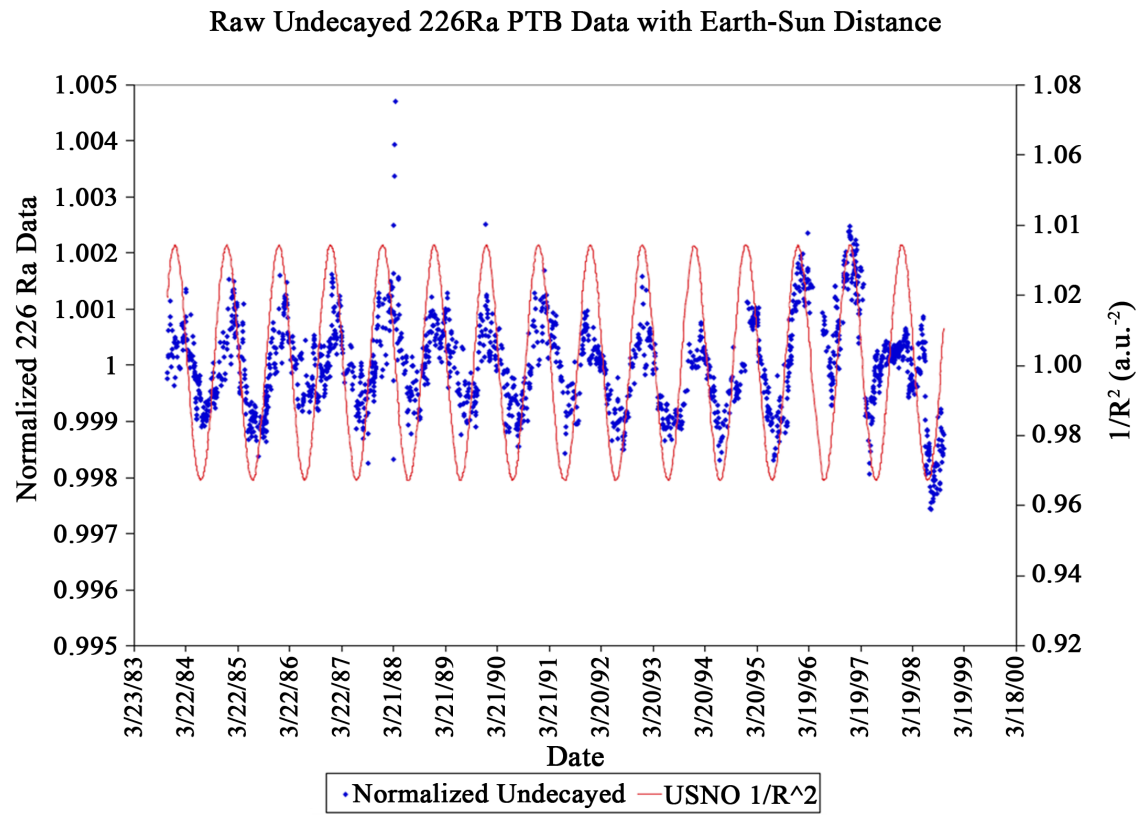

Figure 1. Plot of the ${ }^{226}$ Ra PTB data [1] (15 years) from Fischbach et al. [8] where count rate is plotted vs. time along with $1 / \mathrm{R}_{\text {sun }}{ }^{2}$. The peak of the red curves is about Jan 3 each year while the decay rate appears to peak from early January through late February with a mean about Jan 30 .

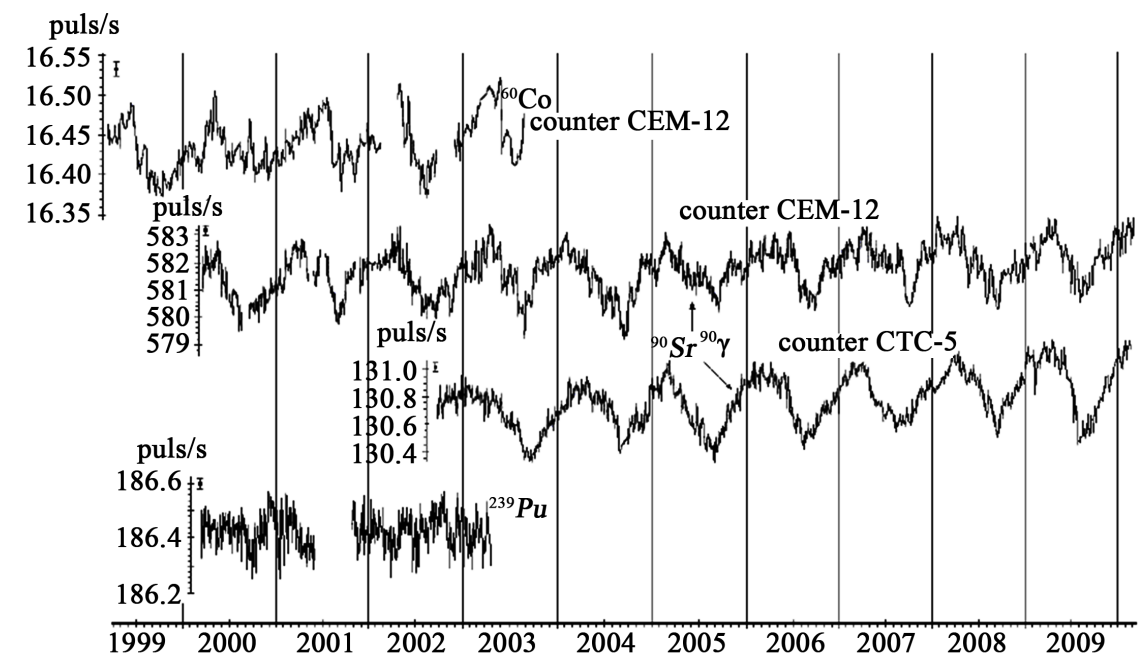

Figure 2. Russian decay data (pulses per second) for ${ }^{60} \mathrm{Co},{ }^{90} \mathrm{Sr}$ and ${ }^{239} \mathrm{Pu} .{ }^{60} \mathrm{Co}$ and ${ }^{90} \mathrm{Sr}$ (beta decay/5 and 20 year half life) showed obvious annual variations while ${ }^{239} \mathrm{Pu}$ (alpha decay/26,000 yr half life) did not. The vertical bars mark Jan 1 of each year. Graph from Parkhomov [6]. 
Table 1. Summary of various tests for time varying nuclear decay including alpha decay, beta minus decay and beta capture. The date of peak decay was somewhat variable with year from about Dec 15 to March 1 even for 10 year continuous experiments of the same radionuclide. The peak-to-valley of the annual modulation varied from $0.0007-0.0037$ of the time average radioactive decay rate.

\begin{tabular}{|c|c|c|c|c|c|c|c|c|c|}
\hline ID \# & Nuclide & Half-life (yr) & Decay Mode & Variation & Technique & $\begin{array}{l}\text { Mag of } \\
\text { Modul }\end{array}$ & $\begin{array}{l}\text { Peak } \\
\text { Day }\end{array}$ & Author & Organization \\
\hline 1 & $226 \mathrm{Ra}$ & 1600 & alpha decay & Annual & ABSOLUTE & $\sim 1.5 \mathrm{E}-3$ & 30 & Siegert [1] & PTB \\
\hline 2 & $154 \mathrm{Eu}$ & 8.59 & beta minus decay & Annual & ABSOLUTE & $\sim 1.5 \mathrm{E}-3$ & 30 & Siegert [1] & РTB \\
\hline $1-2$ & & & & NO & RATIO 1:2 & 0 & NA & Siegert [1] & PTB \\
\hline 3 & $238 \mathrm{Pu}$ & 78.74 & alpha decay & Annual & ABSOLUTE & $\sim 2 \mathrm{E}-3$ & -15 & Ellis [2] & Baylor College \\
\hline 4 & $3 \mathrm{H}$ & 12.3 & beta minus decay & Annual & ABSOLUTE & $3.7 \mathrm{E}-3$ & 45 & Falkenberg [3] & Unknown \\
\hline 5 & $32 \mathrm{Si}$ & 170 & beta minus decay & & & & & Alburger [4] & Brookhaven \\
\hline 6 & $36 \mathrm{Cl}$ & $3.01 \mathrm{E}+05$ & beta minus decay & Annual & RATIO 5:6 & $0.7 \mathrm{E}-3$ & 40 & Alburger [4] & Brookhaven \\
\hline 7 & $54 \mathrm{Mn}$ & $312 \mathrm{~d}$ & beta capture & Annual & ABSOLUTE & $\sim 1 \mathrm{E}-3$ & NA & Jenkins [5] & Purdue \\
\hline 8 & $60 \mathrm{Co}$ & 5.27 & beta minus decay & Annual & ABSOLUTE & $\sim 1.5 \mathrm{E}-3$ & $\sim 90$ & Parkhomov [6] & Moscow State \\
\hline 9 & $90 \mathrm{Sr}$ & 28.9 & beta minus decay & Annual & ABSOLUTE & $\sim 1.5 \mathrm{E}-3$ & $\sim 60$ & Parkhomov [6] & Moscow State \\
\hline
\end{tabular}

2) Hypothesis that only Planck's constant $h$ is in common with strong and weak interactions, and thus might be responsible. Confirm that E \& $M$ tunneling sees an annual variation consistent with $h$ varying across the Earth orbit.

There is very little in common between strong and weak interactions except Planck's constant, so we hypothesized that small variations in Planck's constant might account for the observed synchronized variations in strong and weak decays. If so, then $\mathrm{h}$ would be a maximum on Earth around January-February of each year and a minimum around July-August of each year based on the 20 years of radioactive decay data.

To test the hypothesis that $\mathrm{h}$ was varying across Earth's orbit, a purely electromagnetic experiment was set up to search for the same annual variations. From Jun 14, 2011 to Jan 29, 2014 (941 days), annual variations in tunneling voltage through 5 parallel Esaki tunnel diodes were recorded. Since electron tunneling has both $\mathrm{h}$ in the denominator of the exponent due to tunneling as well as $h^{2}$ in the numerator due to the size of atoms being proportional to $h^{2}$, we set up the experiment with fixed tunnel current and measured the voltage shifts. This had the effect of shifting the temporal variations by 180 degrees compared to current variations at fixed voltage, allowed us to compare our data directly with the radioactive decay plots.

These tunneling variations were similar in magnitude and phase to the radioactive decay variations and supported the hypothesis that there is a gradient in $\mathrm{h}$ across the Earth's orbit. Figure 3 shows the full $2^{1 / 2}$ years of data. It found annual variations of $826 \mathrm{ppm}$ peak-to-valley peaking around Jan 1 .

We next did a best-least-squares fit to the Esaki voltage data with a sine wave to get the following results-an annual $850.5 \mathrm{ppm}$ variation in the tunnel diode current. 
TUNNEL DIODE VOLTAGE VARIATIONS OVER 2 1⁄2 YEARS

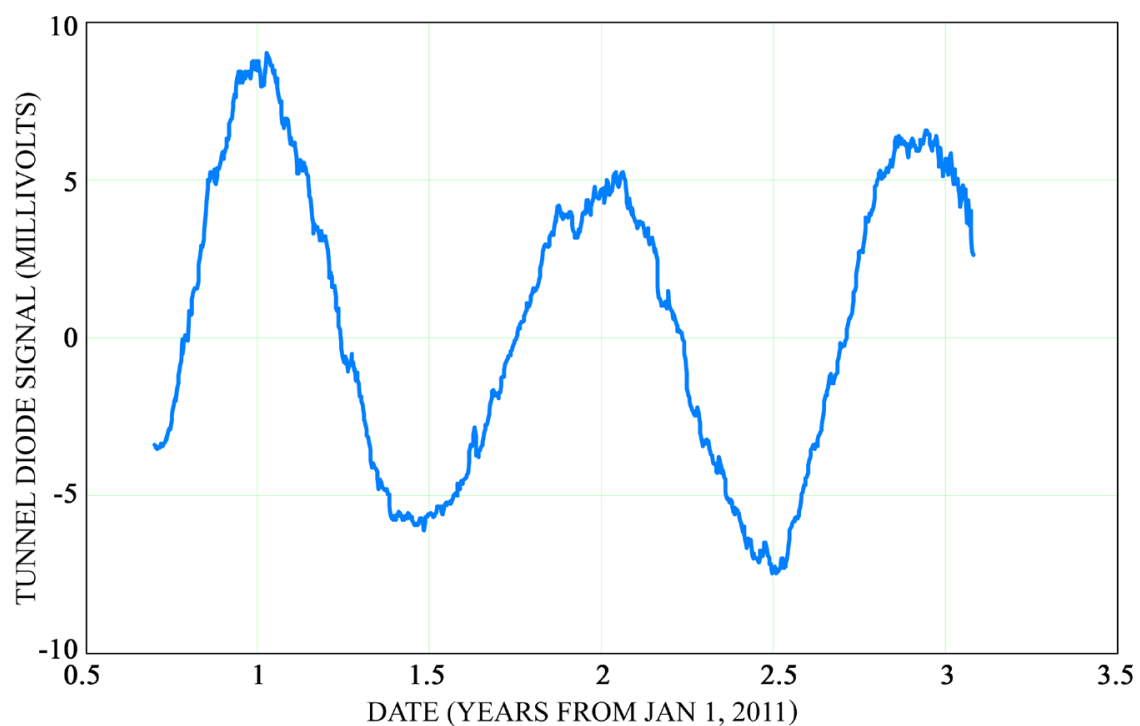

Figure 3. The tunneling voltage data at constant current is plotted here over the $2^{1 / 2}$ year experiment.

Mean Value $=14.97 \mathrm{mV}$, Peak to Valley Sine $=0.01273 \mathrm{mV}$.

Peak to Valley $/$ Mean $=850.5 \mathrm{ppm}$, CosPhase $=-1.273 \mathrm{deg}=$ Dec 31 peak.

Using the estimated $\mathrm{h}$ gain for this tunnel diode of 40.85 , we get an estimated annual variation in Planck's parameter h of $20.8 \mathrm{ppm}$ peak-to-valley.

Please refer to the original paper for more details [11].

3) Why did a recent NIST measurement of h not show variations? [16]

NIST recently did an experiment to measure time variations in $\mathrm{h}$ and found none of significance. However, their experiment was linear, which guarantees that they cannot detect variations in h. The logic is simple. Basically, h controls the time rates of all phenomena. Thus, all their clocks also run slower or faster with $\mathrm{h}$-cancelling out any real variation for a linear process. You must have a nonlinear detection process (such as tunneling or radioactive decay) to measure $\mathrm{h}$ variations.

4) High probability detection of radiation from the sun in the $E$ \& $M$ Planck experiment, creating oscillations in tunnel current locked to day/night (Newly reported here).

In Figure 4 we show blow-ups of the annual and daily peaks over a 2 year period. We note that by choosing $365 \times 2$ days in this Fourier analysis, 365 cycles per year is exactly one cycle per solar day. We also observed a cosmos-locked variation with 366 cycles/year. As is well known, the Earth completes an extra cycle of rotation relative to the outside universe every 365 days. Mathematically there is no cross-coupling between the two Fourier components, which thus represent independent sources of radiation.

Eliminating Temperature Variations as an Explanation: Given that there are daily temperature variations in any lab, we wondered whether those could 

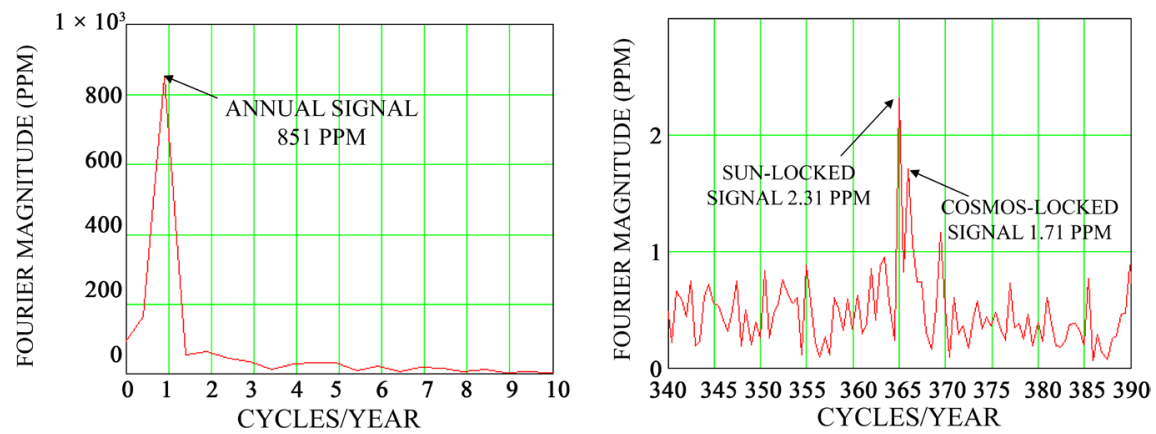

Figure 4. Details of the 2 year Fourier Transform is shown here for the tunnel diode voltage data, which is sampled every 10 seconds ( 3 million times per year) with 10 digits accuracy. The annual, sun-locked and cosmos-locked signals are clearly seen.

create a daily tunnel signal that had not been properly corrected for those temperature variations. Our tunnel diodes were thermally bonded to a thick copper plate that was maintained at $40+/-0.1 \operatorname{deg} \mathrm{C}$ for the full $2^{1 / 2}$ years, and the actual plate temperature was recorded every 10 seconds ( 3 million times a year). We had experimentally calibrated the effect of plate temperature prior to beginning the experiment and removed that small effect in the processing.

Since almost everything happens on the thick Esaki copper plate, which includes the tunnel diodes, temperature sensor and the precision current measurement resistor all bonded to it, we looked carefully at that temperature calibration coefficient which measured the impact of plate temperature on tunnel current. In searching for a more optimal temperature calibration coefficient, we reasoned that uncorrected temperature variations should cause a general increase in the background noise level as well as possibly a one cycle per day signal.

We had experimentally measured the temperature coupling after we set up the experiment prior to gathering annual data, but we knew that there were signs of a slow drift over a few months (Those early months were discarded from the data used in the analysis reported here). Dropping the temperature coupling coefficient by $19 \%$ minimized the noise floor from $91.70 \mathrm{ppm}^{2}$ to $40.44 \mathrm{ppm}^{2}$ and reduced the daily oscillation from about $10 \mathrm{ppm}$ to $2.430 \mathrm{ppm}$. This was obviously a significant coupling, now optimized and removed. Dropping the small quadratic temperature term entirely reduced the noise floor to $40.32 \mathrm{ppm}^{2}$-almost no effect, and dropped the daily oscillation by $2 \%$ to $2.388 \mathrm{ppm}$. Dropping the temperature coefficient for the current by $4 \%$ decreased the noise floor to its minimal value of $40.31 \mathrm{ppm}^{2}$-almost no effect. With all this optimization of temperature correction, the daily oscillation dropped to $2.3138 \mathrm{ppm}$-our final value. This process of trying to adjust our temperature coupling coefficient to minimize the annual signal convinced us that at most a small part of the remaining 24 hour oscillation came from uncorrected temperature variations.

The original temperature correction coefficient was measured when the hardware was first turned on, and we know there was at least a 30-day initial 
burn in transient before stable operation was seen, so it is reasonable that the effective temperature sensitivity could be reduced $19 \%$ from what was first measured. No other temperature coefficients appear to reduce the noise floor significantly. This makes sense because all the key physics happens on the temperature stabilized and temperature monitored Esaki copper plate-which is why we designed it that way.

After all these small corrections for temperature, we got the data shown above in Figure 4, showing both sun-locked and cosmos-locked oscillations. We note that the cosmos locked signals are asynchronous with day-night variations and thus represent a completely different and orthogonal signal-as is normal for different Fourier components.

\section{Probability of False Alarm}

With a complex Gaussian random noise floor, the magnitude ${ }^{2}$ has an exponential distribution with the probability of the noise exceeding a value $\mu$ equal to $\exp (-\mu / \sigma)$. To estimate the rms noise floor $\sigma$ of the magnitude squared we measure the mean and standard deviation of the magnitude ${ }^{2}$ of the complex Fourier transform in regions above and below the signal region, at Fourier components 730 and 732 day. The standard deviations are almost exactly equal, and the means are about $9 \%$ apart.

In Figure 5 we examine the mean value and standard deviation for the squared-magnitude of the signals below and above the yearly peaks. For Complex Gaussian noise, the means and standard deviations should be equal, and this data is close to that. The two portions had similar statistics to about $8 \%$ on the mean value and $3 \%$ on the standard deviation. We took the geometric mean of the two standard deviations-although they were so close to equal that their average gave almost the same value.

Using the familiar exponential model for mean-square complex Gaussian noise, we get a simple exponential probability function as shown in Figure 6 . The probability that the sun-locked signal came from random noise is about $10^{-13}$, and the probability that the cosmos locked signal came from random noise is about $10^{-7}$.

100 Fourier components Below Daily Frequency

$$
\begin{aligned}
& \text { DSubl := submatrix }\left[\overrightarrow{\left[(\mid \text { Data24F })^{2}\right]}, 620,720,0,0\right] \\
& \text { Mean and standard deviation } \\
& \text { from 310-360 cycles/year } \\
& \mu_{1}: \text { mean }(\mathrm{DSub} 1)=0.2084 \mathrm{ppm}^{2} \\
& \sigma_{1}: \text { stdev }(\mathrm{DSub} 1)=0.178923 \mathrm{ppm}^{2}
\end{aligned}
$$

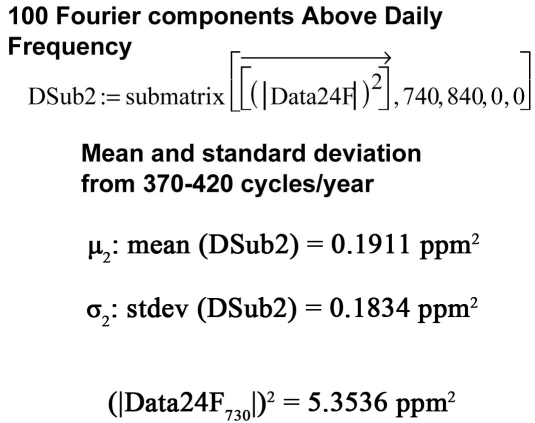

The signal whose probability we are assessing has magnitude-squared of $5.3536 \mathrm{ppm}^{2}$.

Figure 5. Summary of the signal and standard deviation of the noise floor above and below the Sun-Locked signal at pixels 730 days. 


\section{Probability of observed Sun-locked signal by chance

$$
\mathrm{e}^{\frac{-5.3535 \cdot \mathrm{ppm}^{2}}{\sqrt{\sigma_{1} \cdot \sigma_{2}}}}=1.4610 \times 10^{-13}
$$ \\ Probability of observed \\ Cosmos-locked signal by chance

$$
e^{\frac{-2.9249 \cdot \mathrm{ppm}^{2}}{\sqrt{\sigma_{1} \cdot \sigma_{2}}}}=9.7134 \times 10^{-8}
$$

Figure 6. Using the background statistics we computed the probability that the sun-locked peak came from exponential random noise with the given standard deviation of $\left(\sigma_{1} \times \sigma_{2}\right)^{0.5}=0.1811$. The sun-locked peak has about a $10^{-13}$ probability of appearing by random noise, and the cosmos-locked peak has about $10^{-7}$ probability. Such probabilities are usually considered strong support for new phenomena.

\section{Checking the Shape of the Sun-Locked Signal}

To look at the daily response in more detail, we averaged the tunnel data over $365 \times 2=730$ days of 24 hours each. The average is shown in the plot below, Figure 7. This is the voltage required to maintain the constant tunnel current. We note that this residual voltage signal is quite opposite in shape to the effect of lab temperature variations, which tend to be coolest in the morning and warmest in the afternoon, producing more tunneling in the afternoon and thus a lower voltage in our constant current design-the opposite of what we see here.

\section{Checking the Shape of the Cosmos-Locked Signal}

To look at the daily response locked to the cosmos, we averaged tunnel data over $366 \times 2=732$ days shifting the start of the day by $24 \mathrm{hrs} / 366$ days every day. The average is shown in Figure 8, looking rather different than the daily average since it is an independent Fourier component with no coupling to the sun-locked Fourier component. Here the hours match the orientation of the Earth when the data collection began on 10/1/2011.

\section{Discussion}

70 years ago, the vacuum field had been considered to be a simple result of the non-zero ground state energy in quantum mechanics. A large number of experiments, beginning with Casimir [14] [15], have confirmed that the vacuum E \& $M$ fields exist in terawatt $/ \mathrm{cm}^{2}$ intensity, creating up to 19 pounds/inch ${ }^{2}$ pressure on two closely spaced reflective plates. The fields measured are experimentally real and intense.

When annual oscillations in radioactive decay rates were reported by three national labs (US, Russian and German), variations in Planck's constant became a candidate, confirmed in an independent electron tunneling experiment [11]. In addition, that same tunneling experiment with additional analysis showed high confidence detections of Planck radiation coming both from the direction of the Sun and from a direction outside our solar system aligned in right ascension with the red super giant star Betelgeuse. 


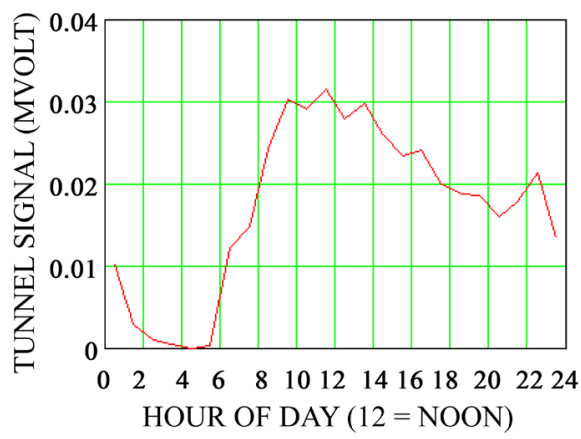

Figure 7. The 24-hour average of the tunnel voltage over 2 years is plotted here. The peak is close to noon as expected. In our experiment the tunnel signal is proportional to $h$.

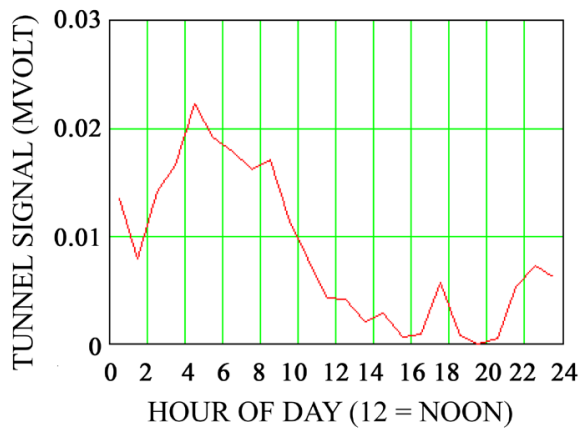

Figure 8. The 24-hour average of the cosmos-locked tunnel current over $365 \times 2$ days is plotted here. The peak response is aligned with 5:30 AM on 10/1/2011, allowing us to search for candidate galactic sources which should be nearly straight overhead at that time. Note that these cosmos-locked and Sun-locked profiles are completely independent of each other since each of the one-hour bins for Sun-centered oscillations spreads out over 48 hours for the cosmos centered oscillation. It is also well known that Fourier components plotted in Figure 4 represent orthogonal signal modes.

This research combined with earlier time varying radioactive decay concludes:

1) The vacuum field is a spectrum of radiation that can vary temporally and spatially to create observable time varying effects when forms of detection are used which are nonlinear in $\mathrm{h}$ (Such as radioactive decay and tunneling).

2) The Sun, and by generalization all stars, is shown to emit a local spectrum of vacuum radiation which adds to the vacuum field from the surrounding universe.

\section{Conclusions}

1) $\mathrm{h}$ is a spectrum of radiation, we call Casimir radiation, that has been shown to vary in intensity and frequency in our laboratories in over 50 experiments.

2) Annual variations in strong decay, weak decay and electromagnetic tunneling are all consistent with $\mathrm{h}$ varying around the orbit of the Earth-peaking in January and minimizing in July. No other cause has been identified.

3) The annual variations of electromagnetic tunnel current were 180 degrees out of phase with strong and weak decay rates, since electromagnetic tunneling has an additional factor of $\mathrm{h}^{2}$ in the numerator of the tunneling exponent due to 
the size of the atoms changing proportional to $h^{2}$. This was predicted and confirmed experimentally by the author, supporting $\mathrm{h}$ as the causal factor.

4) The E \& M tunnel diode data (reported here) detected sun-locked and cosmos-locked variations in tunnel current at 13 sigma and 7 sigma confidence, providing strong evidence that the Sun as well as more distant cosmic sources is generating Casimir radiation.

5) The model that emerges from the above research is that the vacuum field is much more complex and much more interactive than commonly believed. The vacuum field has been proven in 50 years of Casimir lab experiments to vary spatially, temporally and spectrally in our labs. These new data show Casimir radiation also varying across the Earth's orbit, and our own Sun seems to be emitting it as well as a more distant cosmic source.

Future Research: Our conclusion is that the vacuum field is not a single invariant spectrum but is a general background with many local variations, likely with every star emitting it. Such Casimir emissions would tend to increase the value of h over time, making the time rate of the universe speed up with age. For this reason, this process of emitting Casimir radiation from stars may contribute to the observed red shift in the universe-not from a Doppler shift, but simply because time is flowing more slowly in the past with its lower level of Casimir radiation (the vacuum field). Quantitative models for the emission of Casimir radiation would allow evaluation of this option, perhaps also affecting the needs for dark energy and dark matter, which have so far eluded detection.

\section{Conflicts of Interest}

The author declares no conflicts of interest regarding the publication of this paper.

\section{References}

[1] Siegert, H., Schrader, H. and Schötzig, U. (1998) Half-Life Measurements of Europium Radionuclides and the Long-Term Stability of Detectors. Applied Radiation and Isotopes, 49, 1397-1401.

[2] Ellis, K.J. (1990) The Effective Half-Life of a Broad Beam ${ }^{238} \mathrm{Pu} / \mathrm{Be}$ Total Body Neutron Radiator. Physics in Medicine and Biology, 35, 1079-1088. https://doi.org/10.1088/0031-9155/35/8/004

[3] Falkenberg, E.D. (2001) Radioactive Decay Caused by Neutrinos? Apeiron, 8, 32-45.

[4] Alburger, D.E., Harbottle, G. and Norton, E.F. (1986) Half-Life of ${ }^{32}$ Si. Earth and Planetary Science Letters, 78, 168-176. https://doi.org/10.1016/0012-821X(86)90058-0

[5] Jenkins, J.H., et al. (2011) Analysis of Experiments Exhibiting Time Varying Nuclear Decay Rates: Systematic Effects or New Physics? http://arxiv.org/abs/1106.1678

[6] Parkhomov, A.G. (2010) Researches of Alpha and Beta Radioactivity at Long-Term Observations. http://arxiv.org/abs/1004.1761

[7] Towers, S. (2013) Improving the Control of Systematic Uncertainties in Precision Measurements of Radionuclide Half-Life. Applied Radiation and Isotopes, 77, 110-114. https://doi.org/10.1016/j.apradiso.2013.03.003 
[8] Fischbach, E., Jenkins, J.H., Sturrock, P.A., et al. (2011) Evidence for Solar Influences on Nuclear Decay Rates. In: Kostelecky, V.A., Ed., Proceedings of the Fifth Meeting on CPT and Lorentz Symmetry, World Scientific, Singapore, 168-172. https://doi.org/10.1142/9789814327688_0033

[9] Jenkins, J.H., et al. (2009) Evidence for Correlations between Nuclear Decay Rates and the Earth-Sun Distance. Astroparticle Physics, 32, 42-46.

[10] Norman, E.B., Browne, E., Chan, Y.D., Goldman, I.D., Larimer, R.-M., Lesko, K.T., Nelson, M., Wietfeldt, F.E. and Zlimen, I. (1998) Half-Life of ${ }^{44}$ Ti. Physical Review C, 57, 2010-2016. https://doi.org/10.1103/PhysRevC.57.2010

[11] Hutchin, R.A. (2016) Experimental Evidence for Variability in Planck's constant. Optics and Photonics Journal, 6, 124-137. https://doi.org/10.4236/opj.2016.66015 http://www.scirp.org/journal/opj

[12] King, J.A. (2010) Searching for Variations in the Fine-Structure Constant and the Proton-to-Electron Mass Ratio Using Quasar Absorption Lines. Ph.D. Thesis, University of South Wales, South Wales.

[13] Webb, J.K., Flambaum, V.V., Churchill, C.W., Drinkwater, M.J. and Barrow, J.D. (1998) A Search for Time Variation of the Fine Structure Constant. http://arxiv.org/abs/astro-ph/9803165

[14] Casimir, H.B.G. (1948) On the Attraction between Two Perfectly Conducting Plates. Proceedings of the Koninklijke Nederlandse Akademie van Wetenschappen, B51, 793-795.

[15] Bordag, M., Mohideen, U. and Mostepanenko, V.M. (2001) New Developments in the Casimir Effect. Physics Reports, 353, 1-205.

https://doi.org/10.1016/S0370-1573(01)00015-1

[16] Haddad, D., et al. (2017) Measurement of the Planck constant at the National Institute of Standards and Technology from 2015 to 2017. Metrologia, 54, 633-641. https://doi.org/10.1088/1681-7575/aa7bf2 


\section{Appendix-Experimental Hardware Design}

The heart of the experiment is 5 Schottky tunnel diodes wired in parallel and bonded to a $1 \mathrm{~cm}$ thick temperature controlled copper plate, as shown in Figure A1. Heavy duty resistors are bonded to the under-side of the copper plate to control the temperature, which was set to $40 \mathrm{deg} \mathrm{C}$-much higher than the room temperature. A PID thermal controller was setup to maintain the temperature +/- 0.1 deg C. All temperatures, currents, and voltages were monitored every 10 seconds for $2^{1 / 2}$ years-all of them with 10 digit accuracy except for the internal temperature of the voltage and current meters, which were recorded to $0.1^{\circ} \mathrm{C}$ as shown in Figure A2.

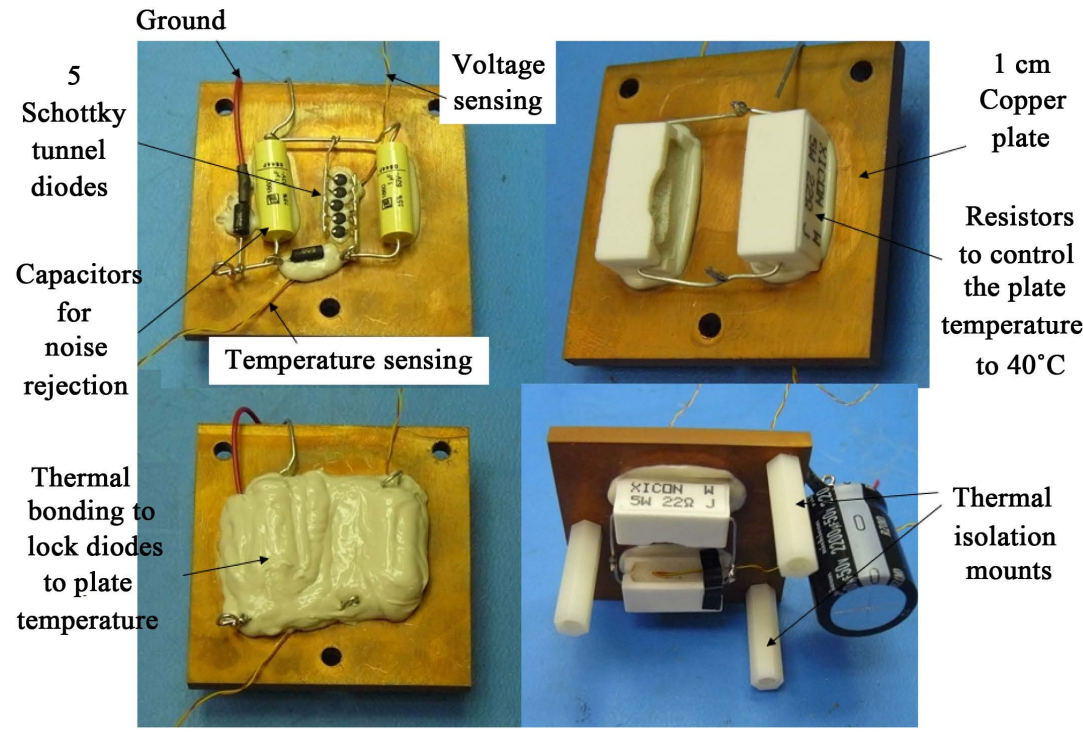

Figure A1. The heart of the tunnel diode experiment is shown here with 5 Schottky tunnel diodes thermally bonded to a thick copper plate, which is temperature controlled to $0.1^{\circ} \mathrm{C}$.

\begin{tabular}{|c|l|c|}
\hline Column & \multicolumn{1}{|c|}{ Data } & Example \\
\hline 0 & Date & $10 / 11 / 2011$ \\
\hline 1 & Time & $12: 00: 10 \mathrm{AM}$ \\
\hline 2 & Current Voltage (mV) & 547.5625365 \\
\hline 3 & Esaki Voltage (mV) & 14.87593562 \\
\hline 4 & Heater element (C) & 67.91888883 \\
\hline 5 & Current Resistor Temp (C) & 39.89013672 \\
\hline 6 & Esaki Plate Temp (C) & 40.01115799 \\
\hline 7 & Shielded Box Temp (C) & 27.96883965 \\
\hline 8 & Esaki Meter Temp (C) & 38.40000000 \\
\hline 9 & Current Meter Temp (C) & 37.80000000 \\
\hline
\end{tabular}

Figure A2. Sample of the data collected every 10 seconds over the 941 day experiment. About 3 million sensor measurements per year for $2^{1 / 2}$ years. 


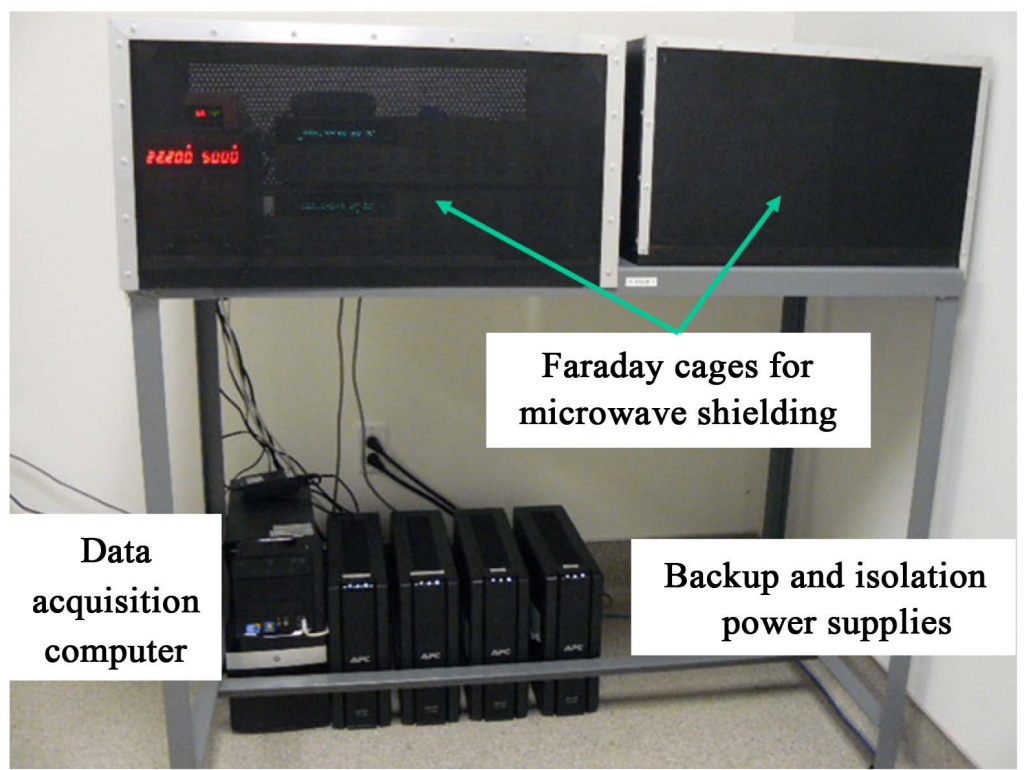

Figure A3. The fully assembled experiment is shown here. Faraday cages were used to protect the experiment from stray microwave and other $\mathrm{E} \& \mathrm{M}$ radiation.

The sensors head and all the electronics were kept in separate Faraday cages to shield them from any local microwave effects, as shown in Figure A3. This additional isolation plus the shielded boxes in which the tunnel diodes were mounted should have isolated these tunnel junctions from all local effects except for the Casimir radiation itself, which we measured. 